\title{
Representation of Radar Image's Timestamp in the Cockpit
}

\author{
Lesheng Hua, Chen Ling, Rickey Thomas \\ University of Oklahoma \\ Norman, OK
}

\begin{abstract}
Data-linked mosaic NEXRAD images can be more than 14 minutes old by the time they reach the cockpit for use by pilots (Elgin \& Thomas, 2004; Novacek et al., 2001; Yuchnovicz et al., 2001). Unfortunately, research has indicated that pilots may not be fully aware of the delay (Yuchnovicz etc., 2001) and that it can adversely affect their decision-making and performance (Chamberlain \& Latorella, 2001). The experiment reported here evaluated the effects of three types of timestamp representation methodologies on participant's accuracy for quick assessments of delay of NEXRAD mosaic radar images. The three types of timestamps investigated were "direct age", "clock" and "UTC" with three levels of delay (short, medium and long). Twenty-one participants compared two radar images, via their timestamps, to determine which one was more recent. The results indicated that "direct age" timestamp resulted in the highest accuracy and fastest response time, and was considered as intuitive and easy to perceive for participants.
\end{abstract}

\section{INTRODUCTION}

The data link service transfers meteorological information to the cockpit via either commercial satellite providers such as XM or WSI, or the ground base Flight Information Service Broadcast (FIS-B) (Vincent et al., 2013). One issue with data-linked radar images in the cockpit is latency. Data-linked weather information can be more than 14 minutes old by the time it reaches the cockpit for use by pilots (Elgin \& Thomas, 2004;

Novacek et al., 2001; Yuchnovicz et al., 2001). Unfortunately, research has indicated that pilots may not be fully aware of the delay or underappreciate the importance of the uncertainty imposed by the delay (Yuchnovicz et al., 2001). Moreover, delayed weather information contributes to navigation decision error (Latorella \& Chamberlain, 2002). The adverse effect of delay on pilot weather decision-making has recently been attributed as a contributing factor in two recent fatal aviation accidents, prompting the National Transportation Safety Board (NTSB) to issue a safety alert to warn pilots that the NEXRAD "age indicator" can be misleading (NTSB, 2011).

Novacek et al. (2001) recommended that more intuitive timestamp designs were needed to alleviate the mental demand necessary and correctly determine the age of NEXRAD images. However, there seems to be little standardization in timestamp methods across radar mosaic images or aviation-weather products in general. Many weather products and websites use UTC form, formatted in "hour: minute: second" to avoid confusion about time zones and daylight saving time. In the Garmin XM weather system, the timestamp consists of a minus symbol and the time, formatted in "-hour: minute." The minus symbol communicates the elapsed time and is supposed to make it easy for users to know the age of the radar information. Another possible representation of time could be through analog display of a clock, which shows current time and observed time with two hands, and highlights the area between the two hands to represent the amount of time delay.

The three types of timestamp described above "direct age", "clock" and "UTC", represent time delay in two different ways- via digital display and analog display. Compared with analog representation, digital displays are more precise and compact (Miller \& Penningroth, 1997). Digital displays have not always proved superior to analog displays (Sander \& McCormick, 1993), particularly when the task requires spatial processing or display values change rapidly.

Another dimension of the problem is the amount of delay that needs to be displayed. Depending on the amount of delay, the mental processes required to gauge the delay might differ among the three timestamps. Thus, in the present study we assess the three proposed timestamp formats under three different delays- short delays, ranging from 1 to 9 minutes, medium delays, ranging from 10 to 19 minutes, and long delays, ranging from 20 to 29 minutes. The goal of the experiment is to determine how best to support fast and accurate judgments concerning the time delay of radar images by users. Our hypothesis is that "direct age" timestamps are the best way to represent the amount of time delay in radar images. 


\section{Participants}

A total of 21 participants took part in this experiment. They are college students recruited from the University of Oklahoma-Norman campus (OU-NC IRB No.0977). The participants were nine men and twelve women. The mean age of participants was 22.6 years $(\mathrm{SD}=2.24)$ and all participants had normal or normalcorrected vision with no color blindness. All gave informed consent and some of them were reimbursed with course credits.

\section{Materials}

Stimuli were displayed on a 20" LCD monitor with resolution of $1600 \times 900$ pixels. Stimuli were a set of screenshots of radar images captured from software-Weather Scope 1.9.3 that was developed by Oklahoma Climatological Survey. The screenshots were $745 \times 568$ pixels. Adobe Photoshop CS2 was used to generate the timestamp on the radar images. The position of timestamp is at a distance of $0.7 \mathrm{~cm}$ from bottom and $2.6 \mathrm{~cm}$ from left side of radar image. The three types of timestamps presented to the participants were direct age, clock, and UTC (see Figure1 (a), Figure1 (b), and Figure1 (c)).

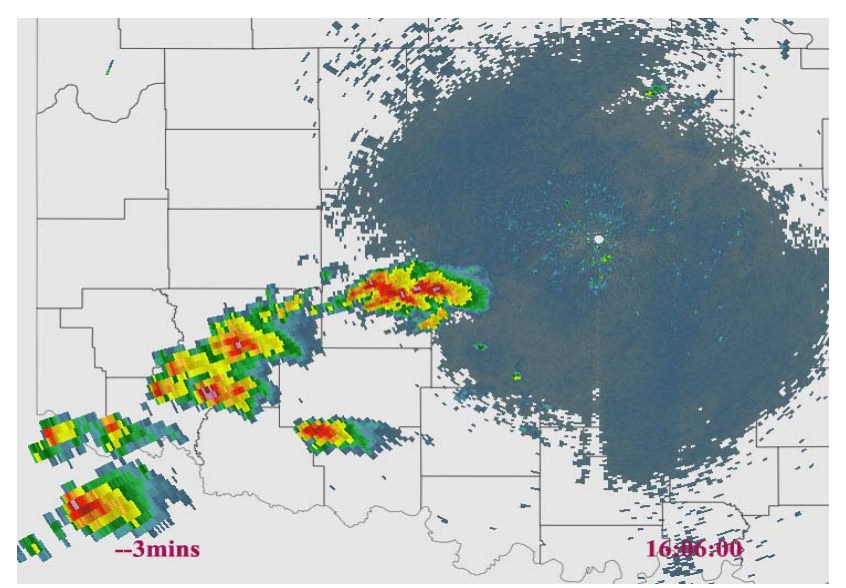

Figure 1(a) "Direct age" timestamp.

The amount of time delay indicated by the timestamp on the left side of the images in Figure1 (a) is three minutes old. The time on the right of the image denotes the current time. The comparison task for participants was to compare "-3mins" with, for example, "-5mins" and determine the "-3mins" timestamp is more recent. Participants could ignore the current time.

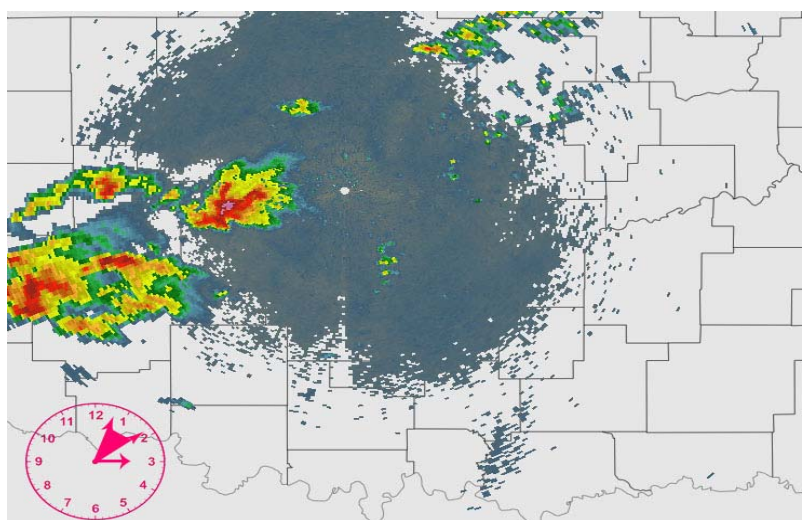

Figure 1(b) "Clock" timestamp

For the Clock timestamp (Figure1 (b)), the two longer hands point to the "minute" and the one shorter hand points to the "hour". The upper hand points to the radar information observed minutes, while the lower hand points to the current minutes. The red area between the two hands indicates the amount of delay. Thus, participants should only need to compare the emergent feature of the size of red areas on the clock face, the smaller area indicating the more recent radar image.

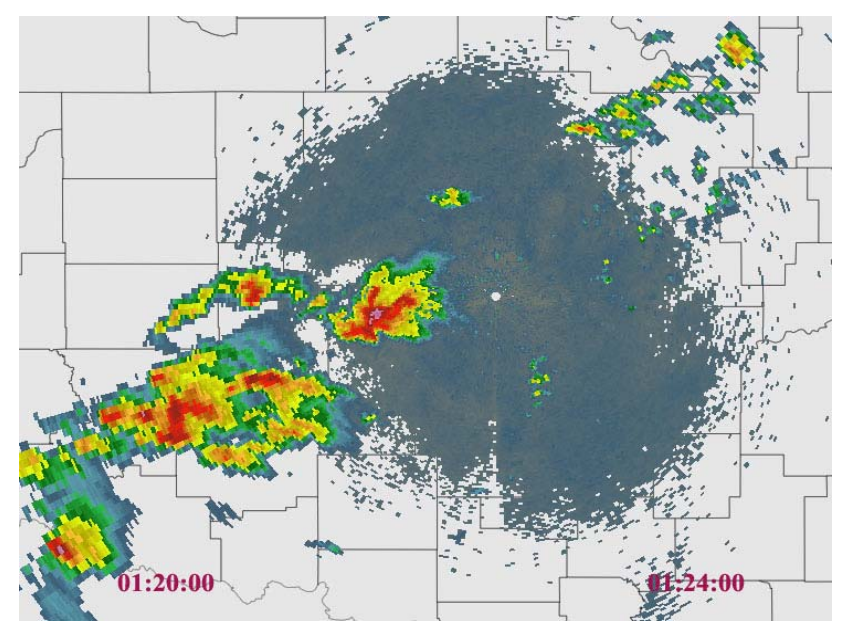

Figure 1 (c) "UTC" timestamp

On the UTC image (Figure 1(c)), the left timestamp indicates the observed time and the right one indicates the current time. The amount of delay can be derived by subtracting the observed time and current time, which is four-minutes delay in this example. To eliminate the mental subtrating process, the current time of the two images with UTC timestamp in the comparison task is the same. Compared with the observed time "01:17:00", for example, "01:20:00" timestamp seems to be more recent.

The independent variables include: types of timestamp (UTC, direct age, clock) and the amount of delay (short (1-10 min.), medium (11-20 min.), long (2130mins). Both independent variables were withinsubject. The dependent variables include: accuracy of 
task and response time. Accuracy is calculated by dividing the number of correct answers by the number of trials for each combination of conditions, which is 15.

\section{Procedure}

Before the experiment, participants gave their consent and filled out the demographic questionnaire. Next, the participants received instruction via a PowerPoint presentation concerning the definitions of timestamp, delay, and the types of the timestamps they would use in the experiment to make comparisons. The experiment interface was programed in Matlab2008b to show a pair of radar images simultaneously with the same timestamp format. The participant's task was to judge which one of the two radar images was more recent based on the timestamp. If participants chose the radar image on the left side, they clicked "more recent" button on the left side and vice versa. The display time was 10 seconds and the display order was random.

\section{RESULTS}

The mean accuracy (percentage correct) and response time among the three types of timestamps at the three levels of time delay are presented in table 1. According to a Repeated measures ANOVA, there was a significant difference among three types of timestamp percent correct $(\mathrm{p}<0.001, \mathrm{~F}(2,19)=11.404)$. Post Hoc tests revealed that the percentage correct of direct age $(\mathrm{M}=93.44, \mathrm{SD}=2.363)$ was significantly higher than the Clock $(\mathrm{M}=74.6, \mathrm{SD}=4.033)$ and UTC $(\mathrm{M}=74.28$, $\mathrm{SD}=3.711$ ) (see Figure 2).

Table 1. Percentage correct (\%) mean (SD.) of three types of timestamp

\begin{tabular}{|l|l|l|l|}
\hline & Direct age & clock & UTC \\
\hline Short & $96.12(9.1)$ & $84.13(28.2)$ & $79.36(18.7)$ \\
\hline Medium & $93.65(11.1)$ & $80.63(19.9)$ & $68.57(19.9)$ \\
\hline Long & $90.48(15.4)$ & $59.05(17.9)$ & $74.92(20.5)$ \\
\hline
\end{tabular}

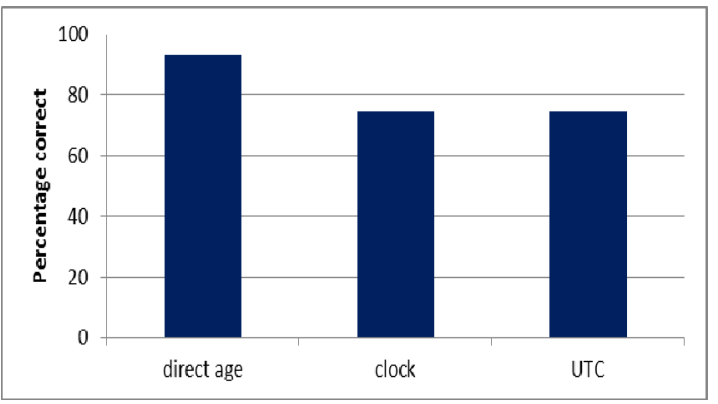

Figure2. Percentage correct of timestamps
The mean response time for the timestamps is plotted in Figure 3. There was a significant difference among three types of timestamp in terms of response time $(p<0.001, F(2,19)=34.89)$. The mean response time for the direct age timestamp $(\mathrm{M}=2.03$ seconds, SD. $=0.137)$ was significantly faster than the Clock $(\mathrm{M}=2.644$ seconds, $\mathrm{SD} .=0.139)$ and UTC $(\mathrm{M}=3.38$ seconds, $\mathrm{SD}=0.146$ ) timestamps.

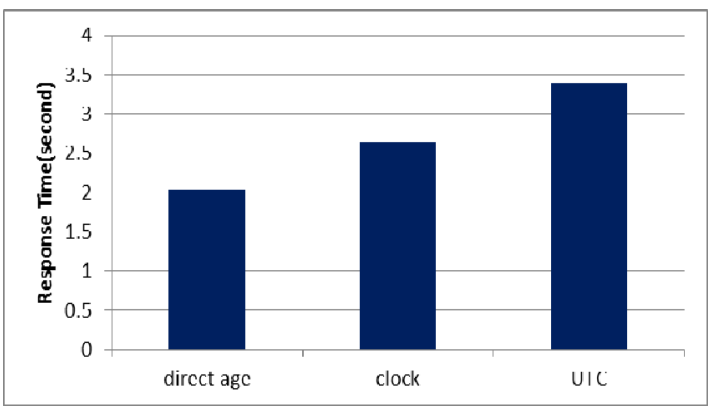

Figure 3. Response time of timestamps

The mean percentage corrects are plotted in Figure 4 for each levels of time delay. The amount of time delay did significantly affect accuracy $(\mathrm{p}<0.001, \mathrm{~F}(2,19)=$ 16.597). The participants exhibited significantly higher accuracy for the short delay $(\mathrm{M}=86.56, \mathrm{SD}=2.446)$ than for the medium $(\mathrm{M}=80.95, \mathrm{SD}=2.298)$ and long delay $(\mathrm{M}=74.81, \mathrm{SD}=2.72)$. We believe that performance was best and response time was fastest for the short time delay (ranged from 1 to 10 minutes) because of the ease of comparing single digital numbers, perceiving the difference in relatively small red areas, or the ease of comparing small digital numbers.

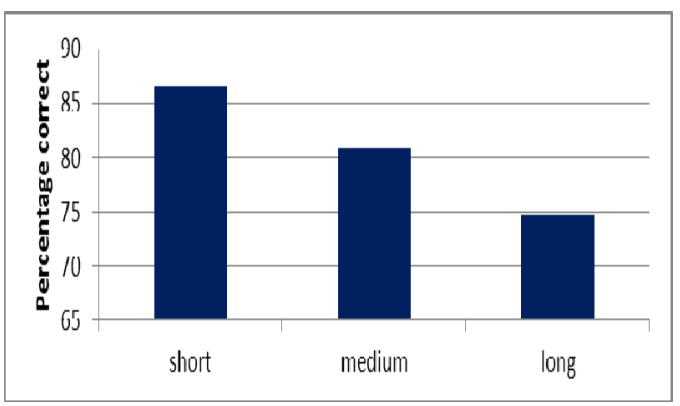

Figure 4. The percentage correct of three levels of time delay

In addition to the main effects, a significant interaction between timestamp types and amount of time delay was found $(\mathrm{F}(4,80)=9.63, \mathrm{p}<0.001)$ for percentage correct (see Figure 5). For the "clock" timestamp, the percentage correct associated with long time delay is relatively worse than accuracy for the other two timestamps. That is, it is difficult for participants to compare relatively large areas on the clock face in the 
analog format. For the "age" timestamp, there is little difference among three levels of time delay.

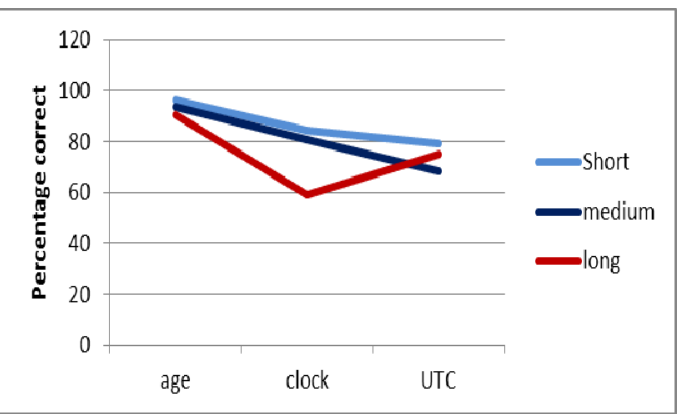

Figure 5. The interaction between types of timestamp and amount of time delay

\section{Discussion}

The experiment was designed to test the prediction that the "direct age" timestamp would be the best methodology to represent time delay. The results of experiment confirmed this expectation since the "direct age" timestamp lead to the most accurate and fastest assessment of delay.

Assessing delay in the "direct age" timestamp only required the understanding of a single number and selecting the more recent image only required the comparison of two numbers. Although the "clock" timestamp only required the perception of area on the clock face, participants found it difficult to compare relatively large areas between two clock faces. The "UTC" timestamp required more mental processing than "direct age", due to the "hour: minute: second" format. Moreover, participants also reported they mentally subtracted between the observed time and current time sometimes to check the amount of time delay. The mental subtraction was unnecessary for the "direct age" condition. So in the limited display time, "UTC" timestamp seems to be not good representation for time delay.

Since the comparison task did not require any special expertise in the interpretation of weather in aviation contexts, although pilots are required to perform many tasks related to the display time, we recommend the "direct age" format as a good method to timestamp weather information in the cockpit. Of course, we did not investigate all possible tasks in this study and are keenly aware that the compatibility between the stimulus, the cognitive processing and the response required for the task(S-C-R compatibility, Wickens et al., 1983) is critically important.

However, in the three timestamp formats that we investigated, the UTC format is the most commonly used one. Through our empirical investigation, we showed that the "direct age" format is more effective in conveying the amount of delay in a delayed display. Our findings can be used to help designers increase awareness of time delay in time sensitive displays.

\section{REFERENCES}

Chamberlain, J. P. \& Latorella, K. A. (2001). Convective weather detection by general aviation pilots with conventional and datalinked graphical weather information sources. Presented at the 20th Digital Avionics Systems Conference October 2001.

Elgin, P. D., \& Thomas, R. P. (2004). An integrated decision-making model for categorizing weather products and decision aids, NASA/TM-2004-212990.

Hansen, J.P. (1997). An Experimental investigation of configural, digital, and temporal information on process displays. Human Factors: The Journal of the Human Factors and Ergonomics Society 1995 37: 539.

Miller, R.J. \& Penningroth, S. (1997). The effects of response format and other variables on comparisons of digital and dial displays. Human Factors, 39(3), 417-424.

National Transportation Safety Board. (2011). Cockpit display factual report. NTSB Number: ERA10MA188.

Novacek, P. F., Burgess, M. A., Heck, M. L. \& Stokes, A. F. (2001). The effect of ownership information and the NEXRAD resolution on pilot decision making in the use of a cockpit weather information display. NASA/CR-2001210845

Sanders, M. S. \& McCormick, E.J. (1993). Human factors in engineering and design. New York, NY: McGraw-Hill, Inc.

Vincent, M., Blickensderfer, E., Thomas, R., Smith, M. \& Lanicci, J. (2013).In-cockpit NEXRAD products: training general aviation pilots. Proceedings of the Human Factors and Ergonomics Society Annual Meeting 2013 57: 81.

Wickens, C.D., Vidulich, M. \& Sandry-Garza, D. (1984). Principles of S-C-R Compatibility with Spatial and Verbal Tasks: The Role of DisplayControl Location and Voice-Interactive Display-Control Interfacing. Human Factors: 
The Journal of the Human Factors and

Ergonomics Society, 26: 533.

Yuchnovicz, D. E., Novacek, P. F., Burgess, M. A.,

Heck, M. L. \& Stokes, A. F. (2001). Use of a

data-linked weather information display and

effects on pilot navigation decision making in a

piloted simulation study. NASA/CR-2001-

211047. 